\title{
Les questions de recherche posées par la Directive Cadre Européenne sur l'Eau: problématique pour les eaux de surface continentales
}

\author{
Research questions arising from the \\ European Water Framework Directive: \\ topics related to inland surface waters
}

\author{
Jean-Gabriel Wasson \\ Cemagref - UR BEA, 3 bis quai Chauveau, BP 220, 69336 Lyon cedex 09 - France \\ jean-gabriel.wasson@cemagref.fr
}

Résumé. - La Directive Cadre Européenne sur l'Eau fixe, à l'horizon 2015, un objectif de "bon état écologique " avec obligation de résultat pour tous les milieux aquatiques naturels. Nous examinons les besoins de recherche dans le domaine des concepts, des méthodes de diagnostic et des modèles prospectifs. Les cours d'eaux sont caractérisés par leur dynamique liée à des processus physiques, chimiques et biologiques qui permettent une certaine réversibilité des perturbations anthropiques. Les indicateurs devraient mettre l'accent sur l'altération des processus. Le bon état se définit comme un écart "léger " à une situation de référence naturelle, mesuré principalement sur des bioindicateurs. Une typologie par hydro-écorégions permet d'identifier des régions homogènes en termes de processus écologiques dominants. Les références seront cherchées dans des bassins où l'anthropisation n'a pas altéré ces processus fondamentaux. Les bioindicateurs devront être régionalisés, harmonisés et inter étalonnés, et de nouvelles métriques (traits biologiques, indices de structure) sont à rechercher. Le diagnostic doit aussi orienter l'action, en hiérarchisant les causes d'altération. Des modèles permettant de prévoir l'efficacité des actions de restauration en terme de réponses biologiques seront nécessaires dans les domaines suivants : morpho-dynamique, relation habitat/peuplements, transfert des polluants, devenir de la matière organique, écotoxicologie, et réponses biologiques complexes tenant compte des interactions entre différents impacts en fonction du contexte naturel.

Mots-clés. - Eaux courantes, Directive Cadre Européenne sur l'Eau, bioindicateurs, modèles, restauration.

Summary - The Water Framework Directive establish for the year 2015 a compulsory goal of "good ecological status" for all natural aquatic ecosystems. We examine the 
research needs to reach this objective for running waters, in the field of concepts, diagnosis methods and predictive models. Running waters are dynamic systems owing to physical, chemical and biological processes that maintain some reversibility towards anthropic perturbations. Ecological indicators should highlight the processes alterations. Good status is defined as a "slight" deviation from natural reference conditions, mainly evaluated from bioindicators. A typology based upon hydro-ecoregions allows the delineation of homogeneous areas as regards dominant ecological processes. Reference will be searched in watersheds where anthropisation did not altered these fundamental processes. Bioindicators should be regionalised, harmonized and intercalibrated, and new metrics (biological traits, structural indices) should be developed. The diagnosis should also hierarchize the causes of ecosystems alteration, in order to orient restoration measures. Models predicting the efficiency of measures expected to restore biological conditions should be developed in the following fields: morpho-dynamics, physical habitat, pollution transfer, organic matter evolution, ecotoxicology, and biological responses to composite alterations interacting in a given natural context.

Key words. - Running waters, Water Framework Directive, bioindicators, models, restoration.

\section{INTRODUCTION}

La gestion par grands bassins fluviaux est une réalité en France depuis la création en 1966 des agences de bassin ; dédiées à l'origine au financement de la lutte contre la pollution, elles sont devenues ensuite des Agences de l'Eau beaucoup plus largement impliquées dans une gestion globale de la ressource et des milieux aquatiques. Avec la loi sur l'eau du 3 janvier 1992 apparaissent dans la législation la notion d'écosystème et un objectif de gestion "équilibrée " devant se traduire par des Schémas Directeurs d'Aménagement et de Gestion des Eaux, les SDAGE. Ceux-ci doivent fixer pour chaque bassin "les orientations fondamentales "d'une gestion équilibrée de la ressource en eau (art. 3), qui doit s'exercer "dans le respect des équilibres naturels" (art. 1), et avec le souci d'assurer "la préservation des écosystèmes aquatiques» (art. 2). Le Cemagref avait contribué à l'époque à une réflexion scientifique pour la mise en œuvre de ces principes (Wasson 1992, 1996a).

La récente Directive Cadre Européenne sur l'Eau (DCEE)" reprend la plupart des concepts qui sous-tendent la gestion de l'eau en France. Dans ce sens, on a pu parler d'un "modèle français", et il est sans

(1) Directive 2000/60/CE du Parlement Européen et du Conseil du 23 octobre 2000 établissant un cadre pour une politique communautaire dans le domaine de l'eau. Adopté le 22 décembre 2000. Site web officiel: http://europa.eu.int/eur-lex/fr/oj/2001/1_32720 $001222 \mathrm{fr} . \mathrm{html}$ 
doute vrai que l'application de cette directive dans notre pays heurtera moins les structures et les habitudes que dans certains autres états membres. Néanmoins, une simple adaptation des textes législatifs ne suffira pas. Les objectifs de la DCEE sont beaucoup plus ambitieux, et la démarche préconisée pose sur plusieurs points une problématique nouvelle pour les gestionnaires. Ceci se traduit au niveau technique par des besoins de connaissance et de méthodes qui interpellent la communauté scientifique, et les outils existants, comme les Systèmes d'Evaluation de la Qualité des cours d'eau (SEQ) (Agences de l'Eau, 1999) devront être adaptés en conséquence.

Le présent article fait le point sur les aspects techniques concernant la mise en œuvre de la DCEE pour les eaux de surface continentales, et évalue les domaines dans lesquels des recherches en hydro-écologie sont à mener. II constitue la synthèse de contributions présentées à diverses occasions (Wasson 2001a et b ; Wasson et al.2001a ; Sachon \& Wasson 2002). Nous privilégions dans ce texte une bibliographie en français qui renvoie à des documents plus complets avec une bibliographie internationale.

\section{LE CONTEXTE DE LA DIRECTIVE CADRE}

Pour les eaux de surface, la directive fixe pour objectif (fig. 1) d'at- teindre à l'horizon 2015 le "bon état " pour tous les milieux naturels, de préserver ceux qui sont en " très bon état ", et d'atteindre le "bon potentiel " dans les milieux fortement artificialisés. II s'y ajoute également un objectif "zéro toxiques", non daté mais clairement affiché. Mais le point essentiel est ici une obligation de résultat dans le délai imparti, et non plus seulement de moyens, la directive fixant seulement un catalogue des mesures possibles qui restent sous la responsabilité des états membres.

Les attendus de ce texte sont particulièrement clairs :

(1) L'eau n'est pas un bien marchand comme les autres mais un patrimoine qu'il faut protéger, défendre et traiter comme tel.

(11)...la politique communautaire de l'environnement doit contribuer à la poursuite des objectifs que constituent la préservation, la protection et l'amélioration de la qualité de l'environnement ainsi que l'utilisation prudente et rationnelle des ressources naturelles, et doit être fondée sur les principes de précaution et d'action préventive et sur le principe de la correction, par priorité à la source, des atteintes à l'environnement, ainsi que sur le principe du pollueur-payeur.

Le bon état (fig. 2) est défini d'après la situation la plus déclassante entre un état chimique se rapportant à des normes de concentration de substances dangereuses 
DCEE - Objectif : “Bon Etat” des eaux

\section{WFD - Goal : "Good status" of waters}

\begin{tabular}{|c|}
\hline Article 4 : Objectifs environnementaux \\
\hline $\begin{array}{l}\text { Obligation de résultat } \\
\text { 1. En rendant opérationnels les programmes de mesures prévus dans le } \\
\text { plan de gestion du district hydrographique: les Etats membres }\end{array}$ \\
\hline $\begin{array}{l}\text { Prévention Immédiate } \\
\text { i) mettent en ceuvre les mesures nćccssaires pour prévenir la } \\
\text { détérioration de l'état de toutes les masses d'eau ... }\end{array}$ \\
\hline $\begin{array}{l}\text { Bon Etat dans } 15 \text { ans } \\
\text { ii) protègent, améliorent et restaurent toutes les masses d'eau de } \\
\text { surface (naturelles) ... afin de parvenir à un bon état des caux de } \\
\text { surface au plus tard quinze ans après la date d'entrćc en vigucur de la } \\
\text { présente directivc, conformément ...à l'annexe } V \text {. sous réserve...(de } \\
\text { dérogations) }\end{array}$ \\
\hline $\begin{array}{l}\text { iii) protègent et améliorent toutes les masses d'eau artificielles et } \\
\text { fortement modifiées, cn vue d'obtenir un bon potenticl écologique et un } \\
\text { bon état chimique des eaux , sous réserve.. (de dérogations...) }\end{array}$ \\
\hline $\begin{array}{l}\text { Suppression des Toxiques } \\
\text { iv) mettent en ouvre les mesures nécessaires... afin de réduire } \\
\text { progressivement la pollution due aux substances prioritaires et } \\
\text { d'arrêter ou de supprimer progressivement les émissions, les rejets et } \\
\text { les pertes de substances dangereuses prioritaires... }\end{array}$ \\
\hline
\end{tabular}

Fig. 1 - Les principaux " objectifs environnementaux " de la Directive Cadre sur l'Eau.

Fig. 1 - The main "environmental objectives" of the Water Framework Directive.

(toxiques) ${ }^{(2)}$, qui seront définies sur des critères écotoxicologiques, et un état écologique qui repose sur une évaluation de la " structure et du fonc-

(2) La DCEE définit comme " dangereuses "les substances "toxiques, persistantes et bioaccumulables". ou "considerées. à un degré équivalent, comme sujettes à caution ". Les substances "prioritaires", qui incluent des substances dangereuses, sont celles qui " présentent un risque significatif pour l'environnement aquatique ", ou " pour l'homme via l'environnement aquatique". La notion de risque combine a la fois le danger intrinseque de la substance et l'étendue de la contamination de l'environnement, fonction des quantités produites ou utilisées. tionnement des écosystèmes aquatiques".

Un objectif aussi ambitieux nécessite une stratégie adéquate en terme de diagnostic et d'actions de restauration. Même si des méthodes opérationnelles existent, pour de nombreux points, et non des moindres, les besoins de recherche et de transfert d'outils de gestion sont patents.

La mise en œuvre de la directive (fig. 3) implique clairement deux phases: d'abord un diagnostic général de l'état des milieux, qui devra préparer la mise en place d'un réseau de 
"Bon Etat" = Etat Chimique + Etat Ecologique

"Good status" = Chemical status + Ecological status

"état écologique" : l'expression de la qualité de la structure et du fonctionnement des écosystèmes aquatiques associés aux eaux de surface, ... classée conformément à l'annexc V;

"bon potentiel écologique" : l'état d'une masse d'eau fortement modifiée ou artificielle, ...

"bon état chimique d'une eau de surface",... l'état chimique requis pour atteindre les objectifs environnementaux fixés à l'article $4, \S 1$, point a), (= bon ètat écologique)

...c'est-à-dire l'état chimique atteint par une masse d'cau de surface dans laquelle les concentrations de polluants ne dépassent pas les nomes de qualité environnementales fixćes à l'annexe IX (= Directives actuelles de valeurs limites d'émission ou concentration de certains toxiques)

et en application de l'article 16, paragraphe $7, \ldots$ (= Normes de concentrations admissibles dans le milieu, à définir sur des critères de toxicité pour le milieu aquatique et l'homme).

Fig. 2 - Définitions de l'état écologique et chimique au sens de la DCEE.

Fig. 2 - Definitions of ecological and chemical status in the WFD.

DCEE : Mise en œuvre

$\Leftrightarrow$ Problématiques de recherche

I - DIAGNOSTIC - délai : 4 à 6 ane
Typologie des milieux et conditions de référence
Evaluation de l'Etat Ecologique
$\rightarrow$ Bioindication

II - RESTAURATION - dèlai : 6 à 15 ans

Identification des causes d'altération :

Relations impacts (multiples) /états

Définition des actions de restauration

Modélisation prospective

Le diagnostic doit orienter l'action...

Fig. 3 - Les principales problématiques de recherche liées à la mise en œuvre de la DCEE.

Fig. 3 - The main research questions arising from the WFD implementation. 
suivi, puis à moyen terme une phase de restauration des milieux qui n'atteignent pas le "bon état ". Les mesures à mettre en œuvre dans ce but seront détaillées dans le premier plan de gestion, prévu en 2009, mais qui devra être précédé d'une large consultation publique. Les principaux éléments de ce plan devront donc être prêts bien avant. Les deux phases sont évidemment liées, car le diagnostic doit orienter les actions de restauration.

Ces deux phases se traduisent par des besoins précis en termes d'outils opérationnels : des méthodes de diagnostic (typologie, bioindicateurs, réseaux de suivi...), et des modèles prévisionnels pour choisir les actions prioritaires de restauration.

Le calendrier est également très serré, puisque la première caractérisation des districts (état des lieux) doit être réalisée en 2004 et actualisée en 2009, les réseaux doivent être opérationnels en 2006, le plan de gestion bouclé en 2009 pour aboutir, sauf dérogations, au bon état des milieux en 2015. Néanmoins, des mises à jour périodiques sont prévues et l'évolution des méthodes est tout à fait envisageable.

\section{LA PHASE DE DIAGNOSTIC}

La phase de diagnostic doit suivre un protocole très précis décrit dans les annexes II et $\mathrm{V}$ de la directive (fig. 4). Elle commence par une typologie des milieux sur des criteres purement physiques, incluant nécessairement un cadre géographique. Dans ces types sont définies des "masses d'eaux ", qui correspondent aux unités à évaluer. Parallèlement, une spatialisation des pressions anthropiques permettra de classer les milieux dans trois catégories :

- les milieux de référence, exempts de contamination toxique, et pour lesquels l'impact des activités humaines est supposé non significatif ;

- les milieux naturels plus ou moins modifiés ou dégradés ;

- les milieux artificiels ou fortement modifiés.

Les milieux de référence, après validation sur le terrain, devront être échantillonnés en vue de définir pour chaque type des " conditions de référence ", notamment biologiques. Ces milieux doivent être dès maintenant préservés de toute dégradation, sauf dérogation à justifier en fonction d'un intérêt général. Ces conditions de référence serviront de repères pour définir "l'état écologique " des milieux naturels, évalué principalement à l'aide de bioindicateurs. Si l'écart à la référence mesuré sur les peuplements est "léger", et siles toxiques ne dépassent pas des seuils qui seront définis sur la base du risque écotoxicologique, les milieux sont considérés en "bon état". Ils devront faire l'objet d'une surveillance régulière et conservés au moins dans cet état.

Dans le cas contraire, si l'écart des peuplements est "modéré à fort" et/ou si les concentrations en toxi- 


\section{DCEE - Mise en œuvre : phase de Diagnostic}

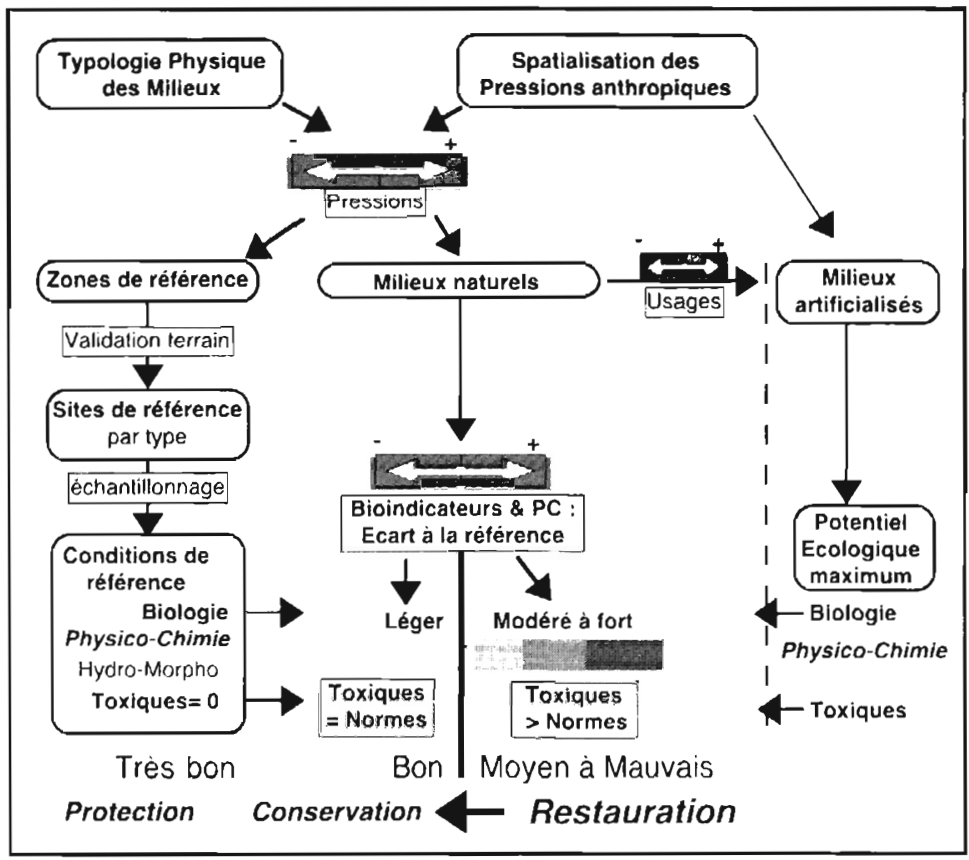

Fig. 4 - Schéma d'organisation de la phase de diagnostic de la DCE

Fig. 4 -Organisation scheme of the WFD initial diagnostic phase.

ques dépassent les seuils fixés, les milieux sont classés dans un état " moyen, médiocre ou mauvais "; ils devront être restaurés pour les ramener au bon état en 2015, sauf dérogations qui devront être soigneusement justifiées en termes de coût et de faisabilité.

Pour les milieux artificiels ou fortement modifiés, les problèmes et les enjeux sont différents. Si la définition des premiers est simple - toute masse d'eau créée par l'homme celle des seconds fait appel à une notion beaucoup plus complexe et leur désignation constituera un premier point-clé pour l'application de la di- rective. Le principe est le suivant : on peut désigner un milieu comme fortement modifié si le fait de restaurer le milieu physique pour atteindre le bon état écologique aurait pour effet de limiter les usages existants, et si ces usages ne peuvent être satisfaits par une meilleure solution environnementale (faisabilité, coût). Cette option doit être justifiée et peut être remise en cause.

L'obligation de bon état chimique s'applique pour tous les milieux artificialisés. Par contre, l'état de référence est remplacé par la notion d'un "potentiel écologique maximum" qu'il serait possible d'atteindre 


\section{Etat Ecologique}

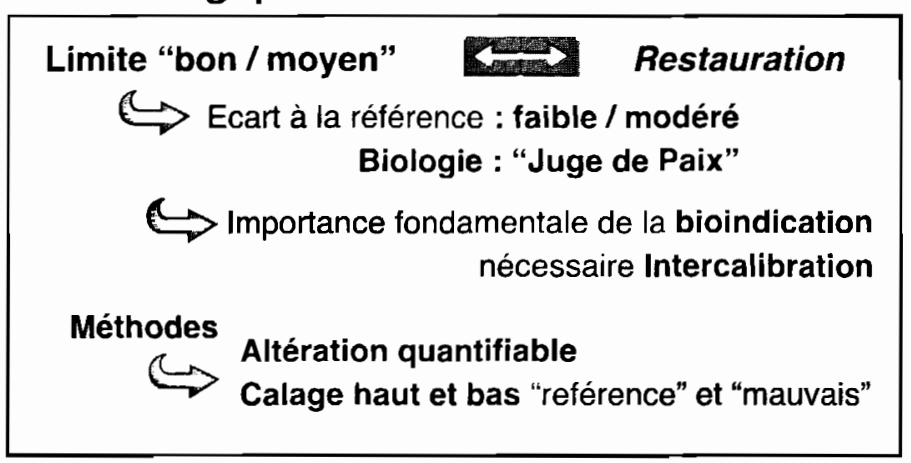

Fig. 5 - Les éléments essentiels de l'évaluation de l'ètat écologique.

Fig. 5 - Fundamental elements for the ecological status evaluation.

compte tenu de l'artificialisation du milieu, et l'objectif à atteindre est le "bon potentiel ", c'est-à-dire un écart léger au potentiel maximum. On voit donc bien les enjeux économiques qui peuvent être liés à la définition du bon potentiel.

\section{1 Évaluation de l'état écologique}

Un deuxième point-clé pour la mise en œuvre de la directive est évidemment la définition de la limite entre les états "bon " et " moyen " (limite vert - jaune sur les cartes), celle qui détermine l'obligation de restauration. II est essentiel de bien comprendre la logique de la démarche: les situations de référence sont déterminées uniquement par analyse des pressions anthropiques, et non pas sur leur richesse biologique puisqu'on admet que celle-ci peut varier selon une typologie naturelle. Un milieu est "de référence" s'il n'est pas, ou très peu, perturbé par l'homme. II ne s'agit pas de conditions "originelles" (en anglais: pristine), mais de situations dans lesquelles l'impact de l'homme sur les écosystèmes est considéré très faible a priori.

Par contre, l'évaluation de l'état écologique repose principalement sur la bioindication : les peuplements sont " juge de paix » (fig. 5) ; peu importe la nature et l'amplitude des modifications des paramètres hydromorphologiques et physico-chimiques: c'est le résultat au niveau des peuplements qui compte en priorité, même si comme on le verra ci-dessous les paramètres physico-chimiques peuvent aussi être déclassants.

D'où l'importance fondamentale de la bioindication, et de l'exercice d'intercalibration (ou inter-étalonnage) qui devra êire réalisé entre les Etats membres sous la houlette de la Commission, pour s'assurer que les " thermomètres" biologiques utilisés ont la même sensibilité (et fiabili- 


\section{Bioindicateurs}

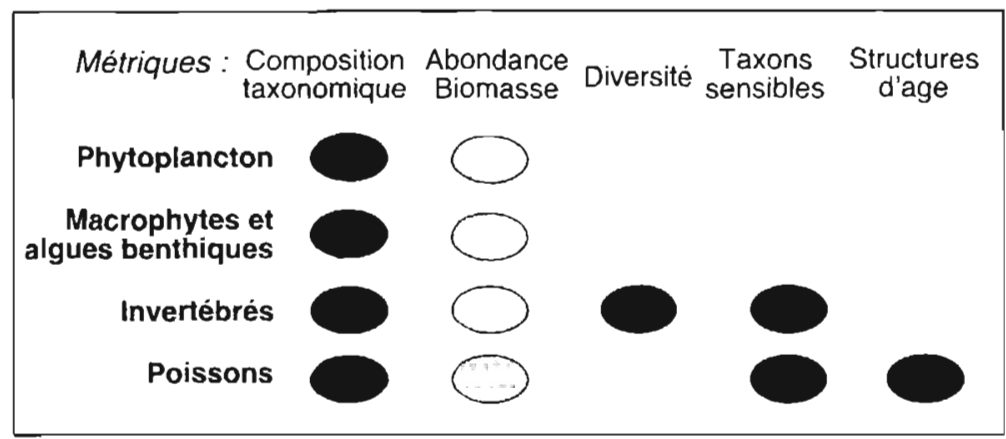

Fig. 6 - Les mètriques de bioindication requises pour l'évaluation de l'état écologique.

Fig. 6 - Bioindication metrics required for the ecological status evaluation.

té). Il faudra ensuite s'accorder sur la limite entre des écarts "léger" et "modéré ", et il semble bien que le seul étalon fiable à ce stade sera, mesuré d'une manière ou d'une autre, une perte de biodiversité taxonomique ou fonctionnelle. Mais dans cet exercice, le calage du thermomètre est aussi important que sa précision, et donc la définition des états biologiques de référence constitue un exercice particulièrement important.

Les bioindicateurs à utiliser reposent sur des métriques qualitatives et quantitatives des peuplements végétaux (phytoplancton, macrophytes et phytobenthos), invertébrés et poissons (fig. 6). Toutefois, il apparaît assez évident que pour les cours d'eau, les invertébrés seront, au moins dans un premier temps, au centre du dispositif car ce sont les bioindicateurs les plus utilisés au niveau européen. Les paramètres d'abondance et de biomasse, très variables en conditions naturelles, devront être utilisés avec précaution ; ils interviendront surtout pour diagnostiquer les proliférations végétales. Les paramètres de diversité des peuplements ne sont explicitement requis que pour les invertébrés, et les structures de population pour les poissons.

Pour l'évaluation des conditions physiques (hydromorphologie), il n'y a pas de normes préconisées. Seuls sont indiqués les compartiments qui devront être évalués pour définir un état de référence, comme par exemple pour les rivières:

Paramètres hydro-morphologiques soutenant les paramètres biologiques

\section{Régime hydrologique}

- quantité et dynamique du débit d'eau

- connexion aux masses d'eau souterraine

Continuité de la rivière (concerne: migration, transfert de sédiments)

\section{Conditions morphologiques}




\title{
Hydro-morphologie
}

\author{
Biologie : "Juge de Paix" $\Rightarrow$ Pas de critères bon / moyen \\ pour l'altération physique \\ Mais $\quad \therefore$ définit les Conditions de Référence \\ essentiel pour la Restauration \\ $\stackrel{\longrightarrow}{\longrightarrow}$ Nécessité d'évaluer l'altération physique
}

Fig. 7 - La place de lhydro-morphologie dans le processus de diagnostic

Fig. 7 - The part of hydro-morphology in the evaluation process.

- variation de la profondeur et de la largeur de la rivière

- structure et substrat du lit

- structure de la rive

Pour les autres etats (bon, moyen...), l'évaluation repose entièrement sur l'impact au niveau des peuplements. Cependant, puisque le diagnostic doit orienter la restauration, il apparaît évident qu'il faudra évaluer les conditions physiques afin de déterminer dans quelle mesure elles contribuent à la dégradation de l'état écologique (fig. 7).

L'évaluation des conditions physico-chimiques est plus complexe, car ces paramètres peuvent être déclassants dans l'évaluation du bon état, ce qui leur confere d'emblée une toute autre importance. Cependant, les normes à appliquer doivent se référer au "fonctionnement de l'écosystème caractéristique ", donc être adaptées aux conditions naturelles pour chaque type de milieu, et aux "valeurs des éléments de qualité biologique ". c'est-à-dire être reliées à l'altération des peuplements.
Là encore, l'évaluation repose sur un lien avec l'impact biologique, mais il n'en reste pas moins qu'une fois les normes définies, un milieu pourra être déclassé du seul fait des parametres physico-chimiques. Ceci permet de tenir compte d'une certaine marge d'incertitude dans la relation entre physico-chimie et bioindicateurs, notamment en ce qui concerne les toxiques dont les effets au niveau des écosystèmes sont encore mal connus. Mais en toute logique, ce cas de figure devrait rester relativement rare; il sera donc nécessaire de bien caler les limites de classes de qualité physico-chimique sur les bioindicateurs, ce qui nécessitera d'affiner par type de milieux les grilles du SEQ Eau (Oudin \& Maupas 2000).

Les paramètres à prendre en compte obligatoirement, toujours pour les rivières, sont les suivants:

Paramètres chimiques et physico-chimiques soutenant les paramètres biologiques

Paramètres généraux

- Température de l'eau

- Bilan d'oxygène 


\section{Physico-chimie}

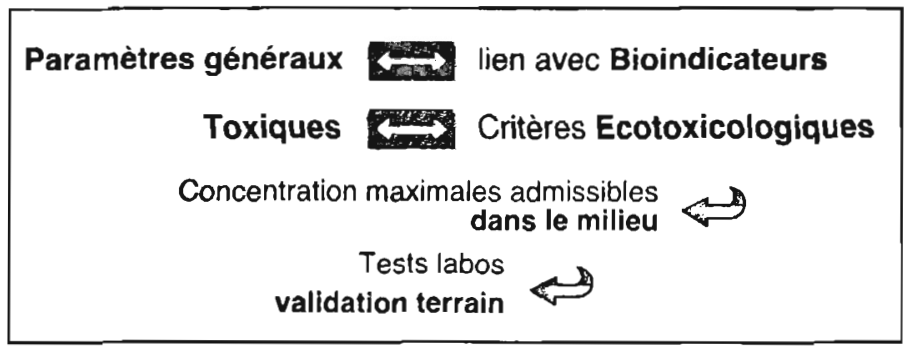

Fig. 8 - Les normes de qualité physico-chimique à relier à des effets biologiques.

Fig. 8 - Chemical quality standards to be related to biological effects.

- Teneur en sel

- État d'acidification

- Concentration en nutriments

\section{Polluants spécifiques}

- Pollution par toutes substances prioritaires recensées comme étant déversées dans la masse d'eau

- Pollution par d'autres substances recensées comme étant déversées en quantités significatives dans la masse d'eau.

Pour les toxiques, les normes de qualité physico-chimique pour le "bon état" sont définies par les états membres sur la base de tests de toxicité aiguë et chronique sur végétaux, micro-crustacés et poissons. Elles doivent définir une concentration moyenne annuelle admissible dans le milieu, pour l'eau, les sédiments ou les organismes. De plus :

- des facteurs de sécurité de 10 à 1000 sont appliqués aux résultats des tests ;

- la bio-accumulation doit être prise en compte ;
- les tests de laboratoire doivent être confrontés à des données de terrain ;

- un examen critique (comité d'expert, publications scientifiques...) et une consultation publique sont demandés.

II y a donc là un domaine de recherche très important pour l'adaptation des normes en fonction des réponses biologiques dans les différents types de milieux (fig. 8).

\section{2 Évaluation de l'état chimique}

Pour l'état chimique, le problème est très différent car il intervient au même niveau que l'état écologique pour définir le "bon état " qui sera déterminé par la situation la plus déclassante. Il s'agit donc d'un point essentiel.

Cet état chimique se réfère dans l'immédiat au respect de normes de qualité environnementales fixées par des directives CEE existantes, et concernant divers produits particulièrement toxiques $(\mathrm{Hg}, \mathrm{Cd}, \mathrm{HCH}$, subs- 
tances dangereuses). Ces normes pourront être modifiées et complétées pour d'autres substances dangereuses dans les années à venir selon des procédures fixées par la directive. L'état chimique se décline en deux catégories (bleu ou rouge) selon que les normes sont respectées ou non.

\section{LA PHASE DE RESTAURATION}

La restauration des milieux, qui constitue l'objectif central de la directive, pose des problèmes scientifiques et techniques beaucoup plus complexes. C'est bien LE point essentiel sur lequel devront porter les recherches en hydro-écologie dans les années à venir. Du fait de l'obligation de résultat, et compte tenu des enjeux économiques et des délais de réponses des systèmes naturels aux actions de restauration, il faudra disposer de modèles prospectifs permettant de prévoir l'efficacité des actions de restauration en terme de réponses biologiques pour choisir la nature et l'intensité des interventions prioritaires. Une réflexion approfondie devrait être menée sur la capacité prédictive des outils existants et la nature des modèles à développer. Ces outils seront dans un premier temps des " modèles d'impact " mettant en relation le niveau d'une variable d'impact (altération physique, pollution organique, eutrophisation, contamination toxique...) avec des réponses biologiques, par exemple:
- Modèles morpho-dynamiques: influence à long terme des modifications des flux hydro-sédimentologiques.

- Modèles d'habitat: réponses biologiques attendues à des modifications hydrologiques et/ou morphologiques.

- Modèles de transfert: cheminement des polluants du bassin à la rivière.

- Modèles d'autoépuration: devenir des flux polluants.

Cependant, deux facteurs viennent compliquer le probleme :

- la variabilité des réponses biologiques à un impact donné en fonction des différents contextes naturels ;

- les interactions entre ces différents types d'impacts.

II faudra donc assez rapidement aboutir à des modèles de réponses fonctionnelles complexes permettant d'évaluer l'importance relative des différents types d'impacts dans un contexte naturel donné.

\section{ORIENTATIONS DE RECHERCHES LIÉES À LA DIRECTIVE}

Ce bref panorama montre que les besoins de recherches portent sur les deux étapes de diagnostic et de restauration, et que les scientifiques devraient s'impliquer à trois niveaux: transfert des connaissances et méthodes existantes, recherche opérationnelle et recherche fondamentale (cf. Wasson 2001a). 


\subsection{Méthodes de diagnostic}

Pour la phase de diagnostic, de nombreuses méthodes sont déjà opérationnelles. Cependant, la démarche imposée par la DCEE à partir des conditions de référence est radicalement différente du système actuel dans lequel les mêmes normes sont appliquées à quelques exceptions près à tous les milieux, comme pour le SEQ Eau. Cela implique une réflexion approfondie sur plusieurs points (cf. Haunia 2002) :

- d'abord sur les concepts : la notion d'état écologique va bien au-delà d'une simple évaluation par paramètre, et devra s'appuyer sur une compréhension des processus qui maintiennent le bon "fonctionnement " du milieu, dont dépendent les structures de peuplement (Wasson 1992, 2001b).

- sur l'approche typologique, en lien avec la définition des conditions de référence, notamment biologiques: il s'agit de proposer une typologie à base physique régionalisée, suffisamment robuste et globale pour constituer un cadre cohérent pour décrire les conditions naturelles abiotiques (hydro-morphologiques et physicochimiques) des différents types, et les peuplements de référence correspondant (Wasson 1996b; Ivol-Rigaut 1998 ; Souchon et al. 2000, Wasson et al. 2001b, 2002). L'ampleur de cette tâche et la rareté des milieux de référence pour certains types impose de passer rapidement d'une approche des- criptive à des modèles de distribution reliés à la typologie physique (Boët \& Fuhs 2000).

- sur les pressions anthropiques : la directive demande d'établir des réseaux de "surveillance " pour les milieux supposés en bon état, et de " contrôle opérationnel " pour les autres. Pour mettre en place ces réseaux, il faut donc définir dans chaque type, pour des milieux sur lesquels l'information est parfois lacunaire (notamment les petits cours d'eau), la probabilité qu'ils n'atteignent pas le bon état dans 15 ans si des mesures spécifiques ne sont pas mises en œuvre ; ceci suppose des modèles "pressions/impacts" spatialisés, et pouvant être reliés à des scénarios d'évolution socio-économique.

- sur les bioindicateurs, qui doivent être normés selon les peuplements de référence et utiliser des métriques permettant l'inter-étalonnage. L'approche la plus simple consisterait à régionaliser les valeurs de référence des indices existants (IBGN) (Ivol-Rigaut 1998 ; Wasson et al. 2002). Cette démarche a déjà été initiée pour I'IBGN dans la cadre du SEQ Bio en cours d'élaboration. Cependant, la diversité des conditions naturelles implique de rechercher de nouvelles métriques qui présentent simultanément les variations les plus faibles selon des facteurs naturels et les plus fortes sur un gradient d'altération. Les paramètres de diversité écologique et les traits biologiques des 
organismes pourront être testés (Usseglio-Polatera et al. 1999 ; Charvet et al. 2000), ainsi que des approches multivariées permettant de s'abstraire de la notion d'indice.

- sur les critères écotoxicologiques permettant d'établir les normes chimiques: la validation terrain des normes qui seront proposées suppose de pouvoir relier des concentrations dans le milieu à des altérations des peuplements (Vindimian 2001), ce qui est déjà rarement le cas, et en fonction des interactions biologiques (complexation, bio-accumulation...), voire des conditions physiques du milieu. II y a là un enjeu majeur pour l'acceptabilité des normes : si elles sont trop sévères, la restauration sera inutilement coûteuse, voire irréaliste; si elles sont trop laxistes, les impacts biologiques persisteront à long terme, empêchant l'atteinte des objectifs.

- sur l'impact écologique des altérations hydro-morphologiques, qui interviennent :

- dans les milieux naturels, en tant qu'élément de diagnostic à coupler nécessairement à la bioindication pour évaluer l'impact relatif des différentes causes d'altération (Wasson et al. 1998 ; Souchon et al. 2002). De ce point de vue, le SEQ Physique en cours d'élaboration reste à caler avec la réponse des peuplements aquatiques.

- dans les milieux fortement modifiés, d'abord pour les définir: la modification physique est-elle in- compatible avec le bon état écologique ? - et si c'est le cas, pour définir le " bon potentiel " : quel état des peuplements peut-on raisonnablement atteindre dans ces milieux?

Concernant ces milieux fortement modifiés, il nous semble qu'actuellement, au niveau français, les enjeux et la difficulté de la démarche ont été largement sous-évalués, ce qui se traduit par une très faible implication des opérationnels et des scientifiques sur ce sujet, lorsque l'on compare avec nos partenaires européens. Cette situation est fortement préoccupante.

\subsection{Modèles prospectifs}

Pour la phase de restauration, il nous semble que très peu d'outils sont actuellement opérationnels ou prêts à être transférés. II y a donc un vrai besoin de recherche fondamentale, non seulement en hydrobiologie sur les concepts, les processus et leurs interactions, les réponses biologiques, mais aussi en socio-économie, à la fois pour rechercher le niveau optimal d'action, et déterminer le coût et l'acceptabilité sociale des mesures correctives. II s'agit de problèmes par nature pluri-disciplinaires (du physique au socio-économique) et multi-échelles (du grand bassin à la molécule).

Il y a là un enjeu majeur pour la recherche dans les dix prochaines années (cf. Menéndez Prieto 2001). Nous ne pouvons à ce stade qu'ébau- 
cher quelques questions structurantes, mais il faut souligner que dans tous les cas, s'impose la nécessité :

- de quantifier l'ampleur des modifications d'origine anthropique par rapport à la variabilité naturelle,

- de spatialiser les problèmes à l'échelle nationale,

- de prévoir leur évolution à 15 ans au minimum,

- d'aboutir à des modèles prospectifs opérationnels,

- de rechercher - selon les attendus de la directive - des solutions à la source, c'est-à-dire au niveau des comportements des acteurs sociaux.

Les principales questions relèvent des thèmes listés ci-après :

\section{Fonctionnement physique des cours} d'eau :

- du bassin versant au tronçon : impact des modifications du paysage, des pratiques culturales, de l'imperméabilisation sur les régimes hydro-sédimentologiques ;

- au niveau des tronçons : les variables de réponse concernent la dynamique hydrologique et morphologique (Hérouin et al. 1995), mais aussi la continuité longitudinale des flux et des migrations, et la connectivité avec le corridor rivulaire (Maridet 1995), en relation avec les modifications des régimes hydro-sédimentologiques et les aménagements structurants (barrages, endiguements, voies navigables...) ou diffus (entretien, chenalisation...) (Wasson et al.
1998 ; Andriamahefa 1999 ; Souchon et al. 2002).

Relations physique - biologie dans les cours d'eau:

- du tronçon à l'habitat aquatique: impact des modifications physiques sur la structure et la dynamique de l'habitat aquatique, recherche de variables intégratives liant la morphologie à l'habitat à l'échelle des tronçons, et conséquences prévisibles sur les différents peuplements aquatiques (et pas seulement les poissons) (Lamouroux et al. 1999 ; Lamouroux et Souchon 2002).

Transfert de polluants diffus (nutriments et toxiques), en fonction du contexte géographique :

- du bassin versant au milieu aquatique : processus de transfert, rôle des pratiques culturales, des structures physiques et végétales, et notamment du corridor de végétation rivulaire (Gril et al. 1999).

Devenir de la matière organique et des nutriments, en fonction des conditions physiques (morphologie et hydrologie) :

- à l'échelle du tronçon et d'un réseau : processus d'évolution de la matière organique et des nutriments, de manière à préciser les relations entre les sources de pollution et les impacts observés dans les milieux.

Devenir et impact des toxiques, en fonction des conditions physiques et de la matière organique : 
- à l'échelle du tronçon et d'un réseau, deux questions majeures restent ouvertes:

- la biodisponibilité des contaminants en fonction de processus physiques, biochimiques et biologiques ;

- les impacts écologiques à court et moyen terme sur les peuplements : comment extrapoler du laboratoire au terrain? de l'individu à l'écosystème ?

\section{Modèles de réponses biologiques} complexes :

- A l'échelle d'un tronçon, comment faire la part des différentes causes d'altérations (physiques, chimiques, toxiques) dans la réponse d'un peuplement? Peut-on hierarchiser ces impacts ? En déduire le niveau de réduction optimal de chacune des causes pour restaurer le " bon état"?

Optimisation des mesures de restauration :

- L'objet de la recherche est alors le " socio-systeme" et son fonctionnement, à des échelles qui vont du territoire d'un SAGE à l'Europe. II s'agit d'un champ très vaste mais essentiel pour atteindre les objectifs de la directive. Parmi les questions ouvertes:

- Quelles sont les structures sociales agissantes (rurales, urbaines, industrielles...) qui génèrent les impacts? Quelles sont les motivations des acteurs?

- Quelle est la perception sociale des changements environnementaux (positifs ou négatifs) ? Quel- les sont les attentes des populations?

- En terme de coût et d'acceptabilité, vaut-il mieux agir au niveau social (changer le comportement des acteurs) ou au niveau technique (ingénierie écologique)? Quelle est la combinaison la plus efficace en terme de résultat?

- Pour changer un comportement, quel est le niveau d'action optimal (local, régional, national, européen...) et sur quels leviers agir (éducation, économie, réglementation...)?

\section{CONCLUSIONS}

En ce qui concerne les eaux de surface, la DCEE ouvre donc, pour les dix années à venir, un champ de recherche assez vaste mais bien délimité ; mais force est de constater que pour l'instant, l'implication des scientifiques au niveau français est relativement faible. L'objectif ambitieux nécessitera pourtant une conjonction des efforts des différents acteurs, parmi lesquels les scientifiques ont un rôle incontournable.

Pour la phase préliminaire de diagnostic, le calendrier serré impose de s'appuyer largement sur les connaissances et les données existantes, et de ce point de vue, les SEQ validés ou en cours d'élaboration constituent certainement un acquis important. Mais un important travail reste à faire pour l'amélioration, la validation et le transfert de méthodes adaptées aux exigences de la directive. En re- 
vanche, pour la phase de restauration qui s'en suivra, le champ de recherche reste totalement ouvert pour le développement de modèles prédictifs permettant d'optimiser les actions à mener, en fonction des différentes conditions naturelles et des contextes socio-économiques. Les délais de mise en œuvre du premier plan d'action, d'ici 2007 à 2009, permettent tout à fait d'engager des programmes de recherche susceptibles de déboucher sur des résultats opérationnels.

De tels programmes nécessitent des structures adaptées. Au niveau national, certains organismes de recherche, comme le Cemagref pour les eaux continentales, occupent de par leurs compétences et leur expérience en matière de transfert de méthodes une position privilégiée. II n'en reste pas moins que des adaptations internes seront nécessaires vu l'ampleur et la durée des actions à mener.

Mais l'effort ne peut reposer sur les seuls organismes de recherche finalisée, ni sur l'échelon national. De ce point de vue, les "Zones Ateliers" du programme "Environnement, Vie et Société " du CNRS (Lévêque et al. 2000), organisées à l'échelle de grands bassins versant comme la Seine, le Rhône, et la Garonne, constituent un cadre particulièrement adapté de par leur approche multiéchelle, la complémentarité disciplinaire des équipes impliquées, et les interactions avec les partenaires opérationnels. A l'échelle de l'Europe, une interconnection sera nécessaire au niveau des structures, et non plus seulement sur des projets labiles, que ce soit au niveau des organismes comme le préfigure le réseau EURAQUA (Menéndez Prieto 2001; Haunia 2002), ou des zones ateliers au sein d'un " Long Term Ecological Research Network". Bien évidemment, un soutien financier conséquent et sur le long terme sera nécessaire pour accompagner cet engagement.

\section{RÉFÉRENCES BIBLIOGRAPHIQUES}

Les références précédées d'un astérisque $\left(^{*}\right)$ sont accessibles en ligne sur le site: http : //www.lyon.cemagref.fr/bea/lhq/lhq.htm sous la rubrique "publications".

Agences de l'Eau, 1999 - Les outils d'évaluation de la qualité des cours d'eau (S.E.Q.). Principes généraux. Études des Agences de l'Eau $N^{\circ} 72$, $13 \mathrm{p}$.

*Andriamahefa H., 1999 - Les hydro-écorégions du bassin de la Loire. Morphologie, hydrologie, pressions anthropiques sur les cours d'eau et les bassins versants (volume1). Thèse de Doctorat Université Jean Monnet Saint Etienne \& Cemagref Lyon BEA/LHQ : 272 p. + annexes

Boët P. \& Fuhs T., 2000 - Predicting presence of fish species in the Seine River basin using artificial neural networks. Artificial neuronal networks. Application to ecology and evolution. S. Lek \& J.F. Guégan ed. : 131-142.

Charvet S., Statzner B., Usseglio-Polatera P. \& Dumont B., 2000 - Traits of benthic macroinvertebrates in semi-natural French streams: an initial application to biomonitoring in Europe. Freshwater Biology, 43 : 277-296.

Gril J.J., Gouy V. \& Carluer N., 1999 Processus de transfert superficiel des 
produits phytosanitaires, de la parcelle au bassin versant. La Houille Blanche, 5-1999: 76-80.

Haunia S., 2002 - Good ecological status: Reference conditions for surface waters. EURAQUA Scientific and technical review n'8, Finnish Environment Institute - SYKE, Helsinki : 99 p.

Hérouin E., Breil P. \& Albert M.B., 1995 Quelles approches pour synthétiser le fonctionnement physique des écosystèmes d'eaux courantes? Bulletin Français de la Pêche et de la Pisciculture, 337/338/339: 141-148.

" Ivol-Rigaut J.M., 1998 - Hydro-écorégions et variabilité des communautés du macrobenthos sur le bassin de la Loire. Essai de typologie régionale et référentiel faunistique. Thèse de Doctorat Université Claude Bernard Lyon I, Cemagref Lyon BEA/LHQ : $271 \mathrm{p}$.

Lamouroux N., Olivier J.M., Persat H., Pouilly M., Souchon Y. \& Statzner B., 1999 - Predicting community characteristics from habitat conditions : fluvial fish and hydraulics. Freshwater Biology, $42: 275-299$.

Lamouroux N. \& Souchon Y., 2002 Simple predictions of instream habitat model outputs for fish habitat guilds in large streams. Freshwater Biology 47(8) : 1531-1542.

Lévêque C., Pavé A., Abbadie L., Weill A. \& Vivien F.D., 2000 - Les zones ateliers, des dispositifs pour la recherche sur l'environnement et les anthroposystèmes. Natures Sciences Sociétés, 4: 44-55.

Menéndez Prieto M., 2001 - Water Modelling as a tool in river basin management within the Framework Directive. EURAQUA Scientific and technical review $n^{\prime 7} 7$, CEDEX : Sección de Edición. Madrid : 198pp.

* Maridet L., 1995 - Rôle des formations végétales riveraines. Recommandations pour une gestion régionalisée. Rapport Cemagref Lyon BEA/LHQ \&
Ministère de l'Environnement, Direction de l'Eau : $31 \mathrm{p}$.

Oudin L.C. \& Maupas D., 2000 - Système d'évaluation de la qualité des cours d'eau. SEQ Eau (version 1). Les études des Agences de l'Eau, N"64. Principes généraux, 21pp. Rapport de présentation, $59 \mathrm{p}$.

Sachon G. \& Wasson J.G., 2002 - La directive Eau de l'Union Européenne: conséquences pour la recherche. $\mathrm{Na}$ tures Sciences Sociétés, 10(Suppl.1) : 93-94.

* Souchon Y., Andriamahefa H., Cohen P., Breil P., Pella H., Lamouroux N., Malavoi J.R. \& Wasson J.G., 2000 Régionalisation de l'habitat aquatique dans le bassin de la Loire. Rapport Cemagref Lyon BEA/LHQ \& Agence de l'eau Loire Bretagne : 291 p.

Souchon Y., Andriamahefa H., Breil P., Albert M.B., Capra H., Lamouroux N., 2002 - Vers de nouveaux outils pour l'aide à la gestion des hydrosystèmes: couplage des recherches physiques et biologiques sur les cours d'eau. Natures Sciences Sociétés, 10(Suppl.1): 26-41.

Usseglio-Polatera P., Thomas S., Beisel J.N. \& Moreteau J.C., 1999 - Illustration de la valeur indicatrice des caractéristiques biologiques des macroinvertébrés d'une communauté benthique à différentes échelles d'observation. Annales de Limnologie, 35(1) : 71-80.

Vindimian E., 2001 - The role of ecotoxicology for monitoring ecosystem health. Freshwater forum, $16: 91-97$.

- Wasson J.G., 1992 - Les orientations fondamentales par bassin: propositions pour une gestion intégrée des écosystèmes d'eau courante. Rapport Cemagref Lyon BEA/LHQ : $32 \mathrm{p}$.

Wasson J.G., 1996a - Les SDAGE : une approche écosystémique. La Houille Blanche, 3-1996 : 23-26. 
Wasson J.G., 1996b - Structures régionales du bassin de la Loire. La Houille Blanche, 6/7-1996 : 25-31.

- Wasson J.G., Malavoi J.R., Maridet L., Souchon Y. \& Paulin L., 1998 Impacts écologiques de la chenalisation des rivières. Cemagref Editions, collection Études, Gestion des Milieux Aquatiques $\mathrm{N}^{\circ} 14: 158 \mathrm{p}$.

Wasson J.G., 2001a - Séminaire " Etat écologique des milieux aquatiques continentaux "Lyon, 20 et 21 mars 2001- Les actes. Cemagref Editions, $187 \mathrm{p}$.

Wasson J.G., 2001b - Objectif 2015 "Bon Etat Ecologique": les enjeux scientifiques et opérationnels de la Directive Cadre Européenne sur l'Eau. Présentation orale, Colloque EDF Hydroécologie 2001: Approche à grande échelle et effets cumulés dans les hydrosystèmes. Chinon, 14-15 juin.

Wasson J.G., Babut M., Dumont B. \& Martinet F., 2001a - L'objectif " bon état écologique": réflexions pour une stratégie de diagnostic à l'échelle d'un grand bassin. Présentation orale, colloque Scientifiques \& Décideurs: Agir ensemble pour une gestion durable des écosystèmes fluviaux; Lyon 7,8,9 juin 2001. Résumé : 85-86.

* Wasson J.G., Chandesris A., Pella H. \& Souchon Y., 2001b - Définition des hydroécorégions françaises. Méthodologie de détermination des conditions de référence au sens de la Directive cadre pour la gestion des eaux. Rapport Cemagref Lyon BEA/LHQ \& Ministère de l'Aménagement du Territoire et de l'Environnement, Direction de l'eau : $68 \mathrm{p}$.

"Wasson J.G., Chandesris A., Pella H. \& Blanc L., 2002 - Définition des Hydro-écorégions françaises métropolitaines. Approche régionale de la typologie des eaux courantes et éléments pour la définition des peuplements de référence d'invertébrés. Rapport Cemagref Lyon BEA/LHQ et Ministère de l'Ecologie et du Développement Durable, Direction de l'Eau: $147 \mathrm{p}$. 